\title{
CLOSED-FORM SUMMATION OF SOME TRIGONOMETRIC SERIES
}

\author{
DJURDJE CVIJOVIĆ AND JACEK KLINOWSKI
}

\begin{abstract}
The problem of numerical evaluation of the classical trigonometric series

$$
S_{\nu}(\alpha)=\sum_{k=0}^{\infty} \frac{\sin (2 k+1) \alpha}{(2 k+1)^{\nu}} \text { and } C_{\nu}(\alpha)=\sum_{k=0}^{\infty} \frac{\cos (2 k+1) \alpha}{(2 k+1)^{\nu}},
$$

where $\nu>1$ in the case of $S_{2 n}(\alpha)$ and $C_{2 n+1}(\alpha)$ with $n=1,2,3, \ldots$ has been recently addressed by Dempsey, Liu, and Dempsey; Boersma and Dempsey; and by Gautschi. We show that, when $\alpha$ is equal to a rational multiple of $2 \pi$, these series can in the general case be summed in closed form in terms of known constants and special functions. General formulae giving $C_{\nu}(\alpha)$ and $S_{\nu}(\alpha)$ in terms of the generalized Riemann zeta function and the cosine and sine functions, respectively, are derived. Some simpler variants of these formulae are obtained, and various special results are established.
\end{abstract}

\section{INTRODUCTION}

\section{Consider the series}

$$
S_{\nu}(\alpha)=\sum_{k=0}^{\infty} \frac{\sin (2 k+1) \alpha}{(2 k+1)^{\nu}} \quad \text { and } \quad C_{\nu}(\alpha)=\sum_{k=0}^{\infty} \frac{\cos (2 k+1) \alpha}{(2 k+1)^{\nu}}
$$

When $\nu>1$, Weierstrass's M-test at once shows that both series converge uniformly for all real values of $\alpha$. The functions $C_{\nu}(\alpha)$ and $S_{\nu}(\alpha)$ are respectively even and odd in $\alpha$, while $C_{\nu}(\alpha)=-C_{\nu}(\pi-\alpha)$ and $S_{\nu}(\alpha)=S_{\nu}(\pi-\alpha)$. Because of these properties, it is sufficient to calculate $C_{\nu}(\alpha)$ and $S_{\nu}(\alpha)$ only over the range $0 \leq \alpha \leq \pi / 2$. In what follows, the particular cases for $\nu=2,3,4, \ldots$ will be separated into $\nu=2 n$ and $\nu=2 n+1$.

Investigation of these series goes back to D. Bernoulli and L. Euler. It is a well-known last-century result, related to Fourier expansion of Euler polynomials $E_{n}(x)$ [1, p. 805, equations 23.1.17 and 23.1.18], that $S_{2 n+1}(\alpha)$ and $C_{2 n}(\alpha)$ are summable in closed form. Moreover, the sums are polynomial in $\alpha$. The series $S_{2 n+1}(\alpha)$ is given by [7, p. 224, equation 14.4.10]

$$
S_{2 n+1}(\alpha)=\frac{(-1)^{n}}{4(2 n) !} \pi^{2 n+1} E_{2 n}\left(\frac{\alpha}{\pi}\right)
$$

Received by the editor September 7, 1993.

1991 Mathematics Subject Classification. Primary 65B10; Secondary 33E20.

Key words and phrases. Summation of series, Riemann zeta function, generalized Riemann zeta function. 
where $0<\alpha<\pi$ for $n=0$ and $0 \leq \alpha \leq \pi$ for $n=1,2,3, \ldots$. Similarly [7, p. 245, equation 17.4.10],

$$
C_{2 n}(\alpha)=\frac{(-1)^{n}}{4(2 n-1) !} \pi^{2 n} E_{2 n-1}\left(\frac{\alpha}{\pi}\right),
$$

where $0 \leq \alpha \leq \pi$ for $n=1,2,3, \ldots$. However, it appears that no simple summation formulae exist for $S_{2 n}(\alpha)$ and $C_{2 n+1}(\alpha)$, which are usually expressed in terms of certain integrals. For example, $S_{2}$ can be replaced by a single integral [7, p. 219, equation 14.2.19]

$$
S_{2}(\alpha)=-\int_{0}^{\alpha / 2} \log (\tan t) d t
$$

or by a combination of integrals [5]. The series $S_{2 n}(\alpha)$ and $C_{2 n+1}(\alpha)$ converge extremely slowly, and the problem of their numerical evaluation has been recently addressed [2, 3, 4]. For the particular case of $n=1$, Dempsey et al. [3] developed a procedure based on Plana's summation formula along with Romberg's method of integration, which significantly improves the convergence and accuracy in comparison with direct summation. Boersma and Dempsey [2] transformed $S_{2 n}(\alpha)$ and $C_{2 n+1}(\alpha)$ into a rapidly convergent series well suited for their computation. In particular, computation for $n=1$ was considered.

We are here concerned with the general case of series in (1) where $\nu>1$. We will show that when $\alpha$ is a rational multiple of $2 \pi$, then $S_{\nu}(\alpha)$ and $C_{\nu}(\alpha)$ can be expressed as finite sums of known constants and values of the generalized Riemann zeta functions of rational arguments. This enables a closed-form evaluation of series $S_{2 n}(\alpha)$ and $C_{2 n+1}(\alpha)$, which are of particular interest.

\section{THE GENERAL CASE}

It is not difficult to verify that the functions defined by

$$
\chi_{\nu}(z)=\sum_{k=0}^{\infty} \frac{z^{2 k+1}}{(2 k+1)^{\nu}}, \quad \operatorname{Li}_{\nu}(z)=\sum_{k=1}^{\infty} \frac{z^{k}}{k^{\nu}}, \quad|z| \leq 1, \nu>1,
$$

are related in the following manner:

$$
\chi_{\nu}(z)=(1 / 2)\left(\operatorname{Li}_{\nu}(z)-\operatorname{Li}_{\nu}(-z)\right)=\mathrm{Li}_{\nu}(z)-2^{-\nu} \operatorname{Li}_{\nu}\left(z^{2}\right) .
$$

In the particular case when $\nu=n=2,3,4, \ldots$ they are known as Legendre's chi-function $\chi_{n}$ of order $n$ [8, p. 283, equation A1.29] and the polylogarithm $\mathrm{Li}_{n}$ of order $n$ [8, p. 282, equation A1.16], respectively. The theory of these and related functions is thoroughly covered in Lewin's standard text [8].

The infinite series $S_{\nu}(\alpha)$ and $C_{\nu}(\alpha)$ in (1) can thus be considered as the imaginary and real parts

$$
S_{\nu}(\alpha)=\operatorname{Im}\left[\chi_{\nu}(\exp (i \alpha))\right], \quad C_{\nu}(\alpha)=\operatorname{Re}\left[\chi_{\nu}(\exp (i \alpha))\right]
$$

of

$$
\chi_{\nu}(\exp (i \alpha))=\mathrm{Li}_{\nu}(\exp (i \alpha))-2^{-\nu} \operatorname{Li}_{\nu}(\exp (i 2 \alpha)) .
$$

Clearly, in order to obtain explicit formulae for the values of $S_{\nu}(\alpha)$ and $C_{\nu}(\alpha)$ at rational multiples of $2 \pi$, it is sufficient to determine such an expression for 
$\operatorname{Li}_{\nu}(\exp (i \alpha))$, where $\alpha=2 \pi p / q$ and $p$ and $q$ are positive integers. We have

$$
\begin{aligned}
\operatorname{Li}_{\nu}(\exp (i 2 \pi p / q)) & =\sum_{k=1}^{\infty} \frac{\exp (k i 2 \pi p / q)}{k^{\nu}} \\
& =\sum_{r=1}^{\infty} \frac{1}{(q r)^{\nu}}+\sum_{r=0}^{\infty} \sum_{s=1}^{q-1} \frac{\exp (s i 2 \pi p / q)}{(q r+s)^{\nu}} \\
& =\frac{1}{q^{\nu}}\left[\sum_{r=1}^{\infty} \frac{1}{r^{\nu}}+\sum_{s=1}^{q-1} \exp (\operatorname{si} 2 \pi p / q) \sum_{r=0}^{\infty} \frac{1}{(r+s / q)^{\nu}}\right],
\end{aligned}
$$

where $k=q r+s$ with $r=0,1, \ldots$ and $s=0,1, \ldots, q-1$, assuming that $r$ and $s$ are not simultaneously zero. Recalling the series representation of the Riemann zeta function [1, p. 807, equation 23.2.1],

$$
\zeta(z)=\sum_{r=1}^{\infty} \frac{1}{r^{z}}
$$

as well as the representation of the generalized Riemann zeta function [10, p. 22]

$$
\zeta(z, a)=\sum_{r=0}^{\infty} \frac{1}{(r+a)^{z}}, \quad a \neq 0,-1,-2, \ldots,
$$

we obtain from (6) that

$$
\operatorname{Li}_{\nu}(\exp (i 2 \pi p / q))=\frac{1}{q^{\nu}}\left[\zeta(\nu)+\sum_{s=1}^{q-1} \exp (\operatorname{si} 2 \pi p / q) \zeta(\nu, s / q)\right] .
$$

Finally, in view of the relationship in $(5 b)$, a similar expression is readily available for $\chi_{\nu}(\exp (i 2 \pi p / q))$, which in conjunction with (5a) leads to

$$
S_{\nu}(2 \pi p / q)=\frac{1}{q^{\nu}} \sum_{s=1}^{q-1} \zeta(\nu, s / q)\left[\sin (s 2 \pi p / q)-\frac{\sin (s 4 \pi p / q)}{2^{\nu}}\right]
$$

and

$$
\begin{aligned}
C_{\nu}(2 \pi p / q)=\frac{1}{q^{\nu}}\{ & \zeta(\nu)\left(1-\frac{1}{2^{\nu}}\right) \\
& \left.+\sum_{s=1}^{q-1} \zeta(\nu, s / q)\left[\cos (s 2 \pi p / q)-\frac{\cos (s 4 \pi p / q)}{2^{\nu}}\right]\right\} .
\end{aligned}
$$

Formulae (8) in general solve the problem of evaluating the series $S_{\nu}(\alpha)$ and $C_{\nu}(\alpha)$ in (1) where $\nu>1$, when $\alpha$ is equal to a rational multiple of $2 \pi$. Here, $p$ and $q$ are positive integers, and no restrictive condition on their greatest common divisor has been assumed. However, when formulae (8) are applied in practice, it is usually preferable that the sums involve as few terms as possible. This can be achieved by choosing $p$ and $q$ in (8) without any common divisors. Further, in view of the symmetry properties of $S_{\nu}(\alpha)$ and $C_{\nu}(\alpha)$ mentioned in the Introduction, it is sufficient to let $\alpha$ vary between 0 and $\pi / 2$. However, when $p$ is odd and $q$ even $(q=2 Q)$, the expressions 
in (8) can be simplified by deriving them starting from the first equality in (4). We then arrive at the particularly simple expressions

$$
S_{\nu}(\pi p / Q)=\frac{1}{(2 Q)^{\nu}} \sum_{s=1}^{Q} \zeta(\nu,(2 s-1) / 2 Q) \sin ((2 s-1) \pi p / Q)
$$

and

$$
C_{\nu}(\pi p / Q)=\frac{1}{(2 Q)^{\nu}} \sum_{s=1}^{Q} \zeta(\nu,(2 s-1) / 2 Q) \cos ((2 s-1) \pi p / Q)
$$

\section{SOME SPECIAL SUMMATION FORMULAE}

The application of (8) and (9) clearly enables us not only to perform a significantly easier computation of the series $S_{\nu}(\alpha)$ and $C_{\nu}(\alpha)$ at rational multiples of $2 \pi$, but also to deduce a number of special closed-form summations. First, it is of interest to check the validity of (8) and (9) by specializing and comparing them with known results. The formulae in (9) give

$$
S_{\nu}(\pi / 2)=\sum_{k=0}^{\infty}(-1)^{k} \frac{1}{(2 k+1)^{\nu}}=2^{-2 \nu}(\zeta(\nu, 1 / 4)-\zeta(\nu, 3 / 4))
$$

and

$$
C_{\nu}(0)=-C_{\nu}(\pi)=\sum_{k=0}^{\infty} \frac{1}{(2 k+1)^{\nu}}=\left(1-2^{-\nu}\right) \zeta(\nu),
$$

which readily follow from well-known results in the theory of the generalized Riemann zeta function $([10$, p. 22$]$ and $[1$, p. 807 , entry 23.2 .20$])$. The special case of $(10)$ when $\nu=2,3,4, \ldots$ can be found in Hansen [7, p. 120, equation 6.3.33 with $s=2 n$, and p. 118 , equation 6.3 .7 with $s=2 n+1]$.

Novel examples of closed-form summations of the series in (1) obtained by the application of formulae in (9) are

$$
S_{\nu}(\pi / 3)=(1 / 2) \sqrt{3} 6^{-\nu}(\zeta(\nu, 1 / 6)-\zeta(\nu, 5 / 6)),
$$

(11b) $C_{\nu}(\pi / 3)=6^{-\nu}\left(1-2^{\nu}\right) \zeta(\nu)+(1 / 2) 6^{-\nu}(\zeta(\nu, 1 / 6)+\zeta(\nu, 5 / 6))$

$$
=(1 / 2)\left(1-3^{-\nu+1}\right) C_{\nu}(0)=(1 / 2)\left(1-3^{-\nu+1}\right)\left(1-2^{\nu}\right) \zeta(\nu),
$$

$$
\begin{aligned}
S_{\nu}(\pi / 4)=(1 / \sqrt{2}) 8^{-\nu}[(\zeta(\nu, 1 / 8) & -\zeta(\nu, 7 / 8)) \\
& +(\zeta(\nu, 3 / 8)-\zeta(\nu, 5 / 8))],
\end{aligned}
$$

(11d) $C_{\nu}(\pi / 4)=(1 / \sqrt{2}) 8^{-\nu}[(\zeta(\nu, 1 / 8)+\zeta(\nu, 7 / 8))$

$$
\begin{gathered}
-(\zeta(\nu, 3 / 8)+\zeta(\nu, 5 / 8))] \\
=(1 / \sqrt{2}) 8^{-\nu}\left[\zeta(\nu) 2^{2 \nu}\left(1-2^{\nu}\right)+2(\zeta(\nu, 1 / 8)-\zeta(\nu, 7 / 8))\right],
\end{gathered}
$$

(11e) $S_{\nu}(\pi / 5)=10^{-\nu}\left[q_{-}(\zeta(\nu, 1 / 10)-\zeta(\nu, 9 / 10))\right.$

$$
\begin{array}{r}
\left.+q_{+}(\zeta(\nu, 3 / 10)-\zeta(\nu, 7 / 10))\right], \\
q_{ \pm}=(1 / 4) \sqrt{10^{ \pm} 2 \sqrt{5}},
\end{array}
$$




$$
\begin{gathered}
C_{\nu}(\pi / 5)=A+10^{-\nu}\left[r_{+}(\zeta(\nu, 1 / 10)+\zeta(\nu, 9 / 10))\right. \\
\left.-r_{-}(\zeta(\nu, 3 / 10)+\zeta(\nu, 7 / 10))\right] \\
r_{ \pm}=(1 / 4)(1 \pm \sqrt{5}), \quad A=10^{-\nu}\left(1-2^{\nu}\right) \zeta(\nu),
\end{gathered}
$$

$(11 \mathrm{~g}) C_{\nu}(\pi / 6)=(1 / 2) \sqrt{3} 12^{-\nu}[(\zeta(\nu, 1 / 12)+\zeta(\nu, 11 / 12))$

$$
-(\zeta(\nu, 5 / 12)+\zeta(\nu, 7 / 12))] .
$$

The expression for $S_{\nu}(\pi / 6)$ obtained by using (9a) is somewhat more complicated, but it is not difficult to verify that it leads to the much simpler result

$$
\begin{aligned}
S_{\nu}(\pi / 6) & =(1 / 2)\left(1+3^{-\nu+1}\right) S_{\nu}(\pi / 2) \\
& =(1 / 2) 2^{-2 \nu}\left(1+3^{-\nu+1}\right)(\zeta(\nu, 1 / 4)-\zeta(\nu, 3 / 4)) .
\end{aligned}
$$

The simplifications in $(11 \mathrm{~b}),(11 \mathrm{~d})$, and $(11 \mathrm{~h})$ rely on the fundamental properties of $\zeta(\nu, a)$ and on the formula [11, p. 658, equation 64:7:10]

$$
\sum_{k=1}^{m} \zeta\left(z, \frac{k}{m}\right)=m^{z} \zeta(z)
$$

\section{4. $S_{2 n}$ AND $C_{2 n+1}$ at RATIONAL MUltiples of $\pi$}

It is obvious that the expressions in (7), (8), and (9) when $\nu=2,3,4, \ldots$ could be rewritten in terms of the polygamma function $\psi^{(n)}$, since there exists the following relation between the generalized Riemann zeta function

$$
\psi^{(n)}(z)=(-1)^{n+1} n ! \zeta(n+1, z), \quad n=1,2,3, \ldots,
$$

and the derivatives $[1$, p. 260 , equation 6.4 .10$]$

$$
\psi^{(n)}(z)=(-1)^{n+1} n ! \sum_{r=0}^{\infty} \frac{1}{(r+z)^{n+1}}
$$

of the digamma function $\psi$. Then the expression in (7) is in fact a polylogarithm of order $n$. We note that our particular case is identical with that obtained by Lewin [9] in his derivation of the expression for the values of the generalized Clausen function.

By employing $\psi^{(n)}$, some further simplifications are possible. For example, we may see how each particular case (8) and (9) can be transformed so as to contain ultimately as few terms as possible. For this purpose, use can be made of (12a) and the following properties of polygamma functions: the reflection formula [1, p. 260, equation 6.4.7]

$$
\psi^{(n)}(1-z)+(-1)^{n+1} \psi^{(n)}(z)=(-1)^{n} \pi[\cot (\pi z)]^{(n)}
$$

and the multiplication formula [1, p. 260, equation 6.4.8]

$$
\psi^{(n)}(m z)=\frac{1}{m^{n+1}} \sum_{k=0}^{m-1} \psi^{(n)}\left(z+\frac{k}{m}\right) .
$$

In order to illustrate this, we have found some additional special sums:

$$
S_{2}(\pi / 2)=G
$$




$$
\begin{gathered}
S_{2}(\pi / 3)=(5 \sqrt{3} / 36)\left[\zeta(2,1 / 3)-2 \pi^{2} / 3\right], \\
S_{2}(\pi / 4)=(1 / 32)\left[\sqrt{2} \zeta(2,1 / 8)-2(\sqrt{2}+1) \pi^{2}-16 \sqrt{2} G\right], \\
S_{2}(\pi / 5)=(1 / 100)\left\{\zeta(2,1 / 5) b_{+}+\pi^{2}\left[(2 / \sqrt{5}) a_{+}-a_{-}\right]\right. \\
\left.-\zeta(2,2 / 5) b_{-}\right\}, \\
a_{ \pm}=\sqrt{10 \pm 2 \sqrt{5}}, \quad b_{+}=a_{+} \sqrt{5}-(1 / 2) a_{+}, \quad b_{-}=a_{-} \sqrt{5}+(1 / 2) a_{-}, \\
S_{2}(\pi / 6)=\frac{2 G}{3},
\end{gathered}
$$

where $G$ stands for Catalan's constant.

Note that the same values as those in (13) can also be deduced from the special values of the Clausen integral $\mathrm{Cl}_{2}(\theta)$ defined by

$$
\mathrm{Cl}_{2}(\theta)=-\int_{0}^{\theta} \log \left(2 \sin \frac{t}{2}\right) d t
$$

which are given in [6] by observing that there exists the relationship [2]

$$
S_{2}(\alpha)=\mathrm{Cl}_{2}(\alpha)-\frac{1}{4} \mathrm{Cl}_{2}(2 \alpha) \text {. }
$$

\section{BIBLIOGRAPHY}

1. M. Abramowitz and I. Stegun (eds.), Handbook of mathematical functions with formulas, graphs and mathematical tables, Dover, New York, 1972.

2. J. Boersma and J. P. Dempsey, On the numerical evaluation of Legendre's chi-function, Math. Comp. 59 (1992), 157-163.

3. K. M. Dempsey, D. Liu, and J. P. Dempsey, Plana's summation formula for $\sum m^{-2} \sin (m \alpha)$, $m^{-3} \cos (m \alpha), m^{-2} A^{m}, m^{-3} A^{m}$, Math. Comp. 55 (1990), 693-703.

4. W. Gautschi, On certain slowly convergent series occurring in plate contact problems, Math. Comp. 57 (1991), 325-338.

5. M. L. Glasser, The summation of series, SIAM J. Math. Anal. 2 (1971), 595-600.

6. C. C. Grosjean, Formulae concerning the computation of the Clausen integral $\mathrm{Cl}_{2}(\alpha)$, J. Comput. Appl. Math. 11 (1984), 331-342.

7. E. R. Hansen, $A$ table of series and products, Prentice-Hall, Englewood Cliffs, NJ, 1975.

8. L. Lewin, Polylogarithms and associated functions, North-Holland, Amsterdam, 1981.

9. __ Some miscellaneous results, Structural Properties of Polylogarithms (L. Lewin, ed.), Amer. Math. Soc., Providence, RI, 1991, pp. 355-375.

10. W. Magnus, F. Oberhettinger, and R. P. Soni, Formulas and theorems for the special functions of mathematical physics, Springer-Verlag, Berlin, 1966.

11. J. Spanier and K. B. Oldham, An atlas of functions, Hemisphere Publishing, Washington, 1987.

Department of Chemistry, University of Cambridge, lensfield Road, Cambridge CB2 1EW, UNITED KINGDOM

E-mail address: J. Klinowski: jk18@uk. ac .cam.phx 